Fassin, D. (2007) Humanitarianism as a Politics of Life. Public Culture, 19(3): 499-520. Lévi-Strauss, C. (1969) [1949] The Elementary Structures of Kinship. Beacon Press. Mauss, M. (1967) [1925] The Gift. The Form and Reason for Exchange in Archaic Societies. W.W. Norton \& Company.

Parry, J. (1986) The Gift, the Indian Gift and the "Indian Gift." Man, 21: 453-473.

Weiner, A.B. (1980) Reproduction: A Replacement for Reciprocity. American Ethnologist, $7(1): 71-85$.

\title{
Global Health
}

Global health can be defined as "collaborative international research and action for promoting health for all" in an equitable manner (Beaglehole and Bonita 2010). The roots of global health lie in colonial medicine, and in the 19th-century concept of "international health," which worked to control the spread of epidemics between countries, including between developed and developing countries.

When the World Health Organization (WHO) was formed in 1948, 70 countries were represented; by 2010, there were 193 countries. After some early setbacks because of the wHO's top-down approach, global health priorities were significantly reformulated in 1978 at a conference in Alma-Ata according to the principle of "health for all." This involved access to health education, immunization, disease control, and essential medications (Farmer, Kleinman, Yong Kim and Basilico 2013). However, with the marketization and privatization of health in the 1980s, global health was reframed as a commodity rather than a right, and, alongside national governments, the World Bank, International Monetary Fund and UNICE F became major players in health. The Gates Foundation is now the largest funder for global health.

Severe health inequity remains a burning human rights issue. With the poor bearing the brunt of both preventable ill health and human rights violations in all countries, health advocacy, public health, and humanitarian and social justice concerns have been jointly mobilized by governments, non-governmental organizations, social and political movements, and health activists to address the "pathogenic role of inequity" (Farmer 2003). Humanitarianism operates in global health as organized institutional and government action, as well as a discourse and justification for community and political action.

The AIDS pandemic of the 1980 s and 1990s catalyzed global health as a field. As anti-retroviral treatments transformed a fatal disease to a manageable one 
and linked to the Millennium Development Goals, billions of dollars became available in grants and debt relief in what has been termed global health's "golden age" (Farmer, Kleinman, Yong Kim and Basilico 2013). The United Nations and WHO ambitiously aimed to extend anti-retroviral treatments to millions in low- and middle-income countries, in African countries particularly. The United States (USA) increased funding to AIDS prevention, care, and treatment tenfold between 2000 and 2006. Despite enormous successes in HIV/AIDS prevention and treatment, however, this roll-out was complicated. Some governments, for example in South Africa, ignored medical findings and denied AIDS treatments to stigmatized populations, with tragic consequences (Fassin 2007). Others, such as Pakistan, failed owing to other government priorities and corruption in state bureaucracies.

Global health now encompasses interventions for infectious diseases (e.g. tuberculosis, malaria, Ebola, Zika), unexplained illness, illness prevention and vaccination, maternal and reproductive health, the impact of war, chemical and nuclear spills, and political violence towards health and health services. Ad hoc actions range from fistula and birthing injuries, for example in Niger (Heller 2018), to immunizations; rural health delivery in Haiti and Rwanda (Farmer et al. 2013) to interventions in societies in transition to democracy. Global health involves temporalities of urgency in situations requiring an immediate mobilization of resources, as well as longer-term temporalities in programs to improve health outcomes, and fractured health systems. These are required to be cost-effective and sustainable, conditions that are not always compatible with reducing inequity.

Humanitarian interventions have comprised an array of methodologies and techniques. "Quick fix" approaches apply technological innovations, including mobile health interventions in remote locations, flying doctors and temporary surgical camps, and mass screenings for infectious disease.

Controversies arise concerning the delivery of justice and intervention within colonial and postcolonial matrices of organized and institutional power and knowledge. Interventions must also address challenges involving scaling and translating universalist projects for local contexts. For example, in the Republic of Guinea, humanitarian responses to Ebola, insensitive to and ill informed about local histories of structural violence, unsettled the explosive but latent social tensions in everyday modes of social accommodations between existing burial practices and hospital medicine, local political structures and external political subjection, mining interests and communities, and those suspected of "sorcery" - which severely restricted its efficacy (Fairhead 2016). Responses must also address the application of Western-derived knowledge to local contexts, accommodate tensions between local, indigenous, and 
standardized global approaches, and confront issues of professionalism and legitimacy — as well as local community efforts to resist and decolonize topdown deductive approaches of the global health imperium (Farmer et al. 2013). While historically the "West" has been at the center of economic imperialism and global health, power is fluid and shifting, as evident in China's medical campaigns across 30 African countries, including the building of private hospitals, in postwar and developing contexts.

Despite clinicians' best efforts, citizens of the USA are dying on the streets. This is because of a confluence of inadequate public health care, acute funding pressures on community psychiatry, social inequality, inner-city degeneration, and homelessness and related illnesses (Brodwin 2013). In Europe, the confluence of changing food environments, long-term financial crisis and austerity, and cuts in state health provision have also led to deepening health inequities. Controversies additionally surround the politics of health care provision to asylum seekers. For example, in Europe access to health care for clandestine and illegal migrants varies significantly across countries, raising questions about health equity, global bioethics, and the need for a more joined-up approach for mitigating health risks in some of the world's newest and most vulnerable communities.

\section{Nichola Khan}

\section{References}

Beaglehole, R., Bonita, R. (2010) What Is Global Health? Global Health Action, 3: 5142. Brodwin, P. (2013) Everyday Ethics. Voices from the Front Line of Community Psychiatry. University of California Press.

Fairhead, J. (2016) Understanding Social Resistance to the Ebola Response in the Forest Region of the Republic of Guinea: An Anthropological Perspective. African Studies Review, 59(3): 7-31.

Farmer, P. (2003) Pathologies of Power: Health, Human Rights and the New War on the Poor. University of California Press.

Farmer, P., Kleinman, A., Yong Kim, J., Basilico, M. (2013) Reimagining Global Health: An Introduction. University of California Press.

Fassin, D. (2007) When Bodies Remember: Experiences and Politics of AIDs in South Africa. University of California Press.

Heller, A. (2018) Fistula Politics. Birthing Injuries and the Quest for Continence in Niger. Rutgers University Press. 